EPJ Web of Conferences 66, 03014 (2014)

DOI: $10.1051 /$ epjconf/ 20146603014

(C) Owned by the authors, published by EDP Sciences, 2014

\title{
The ratio method: a new way to look at halo nuclei
}

\author{
P. Capel ${ }^{1, a}$, R. C. Johnson ${ }^{2, b}$, and F. M. Nunes ${ }^{3, c}$ \\ ${ }^{1}$ Physique Nucléaire et Physique Quantique, Université Libre de Bruxelles (ULB), B-1050 Brussels, Belgium \\ ${ }^{2}$ Department of Physics, University of Surrey, Guildford GU2 7XH, UK \\ ${ }^{3}$ National Superconducting Cyclotron Laboratory and Department of Physics and Astronomy, Michigan State \\ University, East Lansing, MI 48824, USA
}

\begin{abstract}
A new reaction observable is presented to study exotic loosely-bound structures, such as halo nuclei. It consists of the ratio of two angular distributions, e.g. one for breakup and one for elastic scattering. This ratio is nearly independent of the reaction mechanism and is very sensitive to the projectile structure. This new ratio method is illustrated on the particular case of ${ }^{11} \mathrm{Be}$, the archetypal one-neutron halo nucleus.
\end{abstract}

\section{Introduction}

The development of radioactive-ion beams (RIB) in the mid-80s, has enabled us to explore the nuclear landscape away from stability. Various exotic structures have been uncovered thanks to this technological breakthrough, among which halo nuclei [1]. These light neutron-rich nuclei exhibit a much larger matter radius than their isobars. This surprising property is understood as resulting from their very low binding energy for one or two neutrons. Thanks to this lose binding, the valence neutrons exhibit a high probability of presence at large distance from the other nucleons. They hence form a sort of halo around a compact core that contains the other nucleons. The best known halo nuclei are ${ }^{11} \mathrm{Be}$ and ${ }^{15} \mathrm{C}$ with a one-neutron halo, and ${ }^{6} \mathrm{He}$ and ${ }^{11} \mathrm{Li}$ with two neutrons in their halo. Albeit possible, proton halos are less probable due to the presence of the Coulomb barrier between the core and the valence protons, which hinder the development of a long tail in the wave function.

Being located on the shoulder of the valley of stability, halo nuclei cannot be studied through usual spectroscopic techniques and one must resort to indirect methods to infer information about their structure. Reactions, such as breakup or elastic scattering are among the most used tools to study halo nuclei [2]. However, to extract valuable information from such experimental data, a good understanding of the reaction process is required. Various reaction models have been developed with this aim, e.g., the Continuum Discretized Coupled Channel method (CDCC), the time-dependent model (TD), and the Dynamical Eikonal Approximation (DEA) (see Ref. [3] for a recent review and Ref. [4] for a comparison of these three models). Theoretical analyses of reactions have shown that higher-order effects such as coupling within the continuum lead to complicated reaction mechanisms that hinder the analysis of reaction cross sections simply in terms of the projectile structure. Moreover,

\footnotetext{
ae-mail: pierre.capel@ulb.ac.be

be-mail: r.johnson@surrey.ac.uk

ce-mail: nunes@nscl.msu.edu
}

This is an Open Access article distributed under the terms of the Creative Commons Attribution License 2.0, which permits unrestricted use, distribution, and reproduction in any medium, provided the original work is properly cited. 
even state-of-the-art reaction models are sensitive to inputs such as the optical potentials used to simulate the scattering of projectile components by the target. The core-target interaction in particular is often unknown as the core is usually itself radioactive.

To circumvent this problem, we suggest a new observable that is nearly independent of the reaction mechanism and that is therefore more sensitive to the projectile structure than usual breakup or elasticscattering cross sections [5,6]. This observable consists of the ratio of angular distributions for two different processes such as breakup and elastic scattering. It can be directly compared to the halo form factor predicted by an adiabatic description of the reaction [7].

After describing the theoretical framework in which reactions are modeled, we introduce this new observable. It is then tested using a fully dynamical model of reactions for the case of ${ }^{11} \mathrm{Be}$ impinging on $\mathrm{Pb}$ at $69 \mathrm{AMeV}$ and $\mathrm{C}$ at $67 \mathrm{AMeV}[2]$. We end this contribution by a brief conclusion and the prospects of this work.

\section{Theoretical framework}

We consider reactions in which a one-neutron halo nucleus is impinging on a target $T$. The projectile is modeled as a valence neutron $\mathrm{n}$ loosely bound to a core $c$. It is described by the Hamiltonian

$$
H_{0}=T_{r}+V_{c \mathrm{n}}(\boldsymbol{r})
$$

where $\boldsymbol{r}$ is the neutron-core relative coordinate, $T_{r}$ is the $c$-n kinetic-energy operator, and $V_{c \mathrm{n}}$ is a potential that simulates the interaction between the halo neutron and the core. The parameters of that potential are adjusted to reproduce the binding energy of the projectile and some of its low-lying levels. The target $T$ is supposed structureless and its interaction with the projectile components is simulated by the optical potentials $V_{c T}$ and $V_{\mathrm{n} T}$. Within this framework, the theoretical study of reactions involving halo nuclei reduces to solving the three-body Schrödinger equation

$$
\left[T_{R}+H_{0}+V_{c T}\left(R_{c T}\right)+V_{\mathrm{n} T}\left(R_{\mathrm{n} T}\right)\right] \Psi(\boldsymbol{r}, \boldsymbol{R})=E_{T} \Psi(\boldsymbol{r}, \boldsymbol{R}),
$$

where $\boldsymbol{R}$ is the projectile-target relative coordinate and $T_{R}$ is the corresponding kinetic-energy operator. The projectile is initially in its ground state $\phi_{0}$ while impinging on the target:

$$
\Psi(\boldsymbol{r}, \boldsymbol{R}) \underset{Z \rightarrow-\infty}{\longrightarrow} e^{i K Z+\ldots} \phi_{0}(\boldsymbol{r}),
$$

where $K$ is the wave number of the initial relative motion of the projectile to the target, in direction $\widehat{Z}$.

\section{The ratio idea}

It has been observed that angular distributions for elastic scattering and breakup exhibit very similar features: Coulomb rainbow, oscillatory patterns etc. [8]. This result can be qualitatively understood within the Recoil Excitation and Breakup model (REB) [7], which relies on two simplifying assumptions: First it includes an adiabatic treatment of the excitation of the projectile. Second, it neglects $V_{\mathrm{n} T}$. Under these two assumptions the three-body problem (2) can be solved semi-analytically and the elastic-scattering cross section can be elegantly factorized (see Ref. [7] for details):

$$
\frac{d \sigma_{\mathrm{el}}}{d \Omega}=\left|F_{0,0}\right|^{2}\left(\frac{d \sigma}{d \Omega}\right)_{\mathrm{pt}} .
$$


In that expression, the cross section for the elastic scattering for a pointlike projectile $(d \sigma / d \Omega)_{\mathrm{pt}}$ is multiplied by a form factor that accounts for the extension of the halo

$$
F_{0,0}=\int\left|\phi_{0}(\boldsymbol{r})\right|^{2} e^{i \boldsymbol{Q} \cdot \boldsymbol{r}} d \boldsymbol{r},
$$

where $Q$ is proportional to the transferred momentum. A similar result can be obtained for breakup

$$
\frac{d \sigma_{\mathrm{bu}}}{d E d \Omega}=\left|F_{E, 0}\right|^{2}\left(\frac{d \sigma}{d \Omega}\right)_{\mathrm{pt}},
$$

where the form factor reads

$$
\left|F_{E, 0}\right|^{2}=\sum_{l j m}\left|\int \phi_{l j m}(E, \boldsymbol{r}) \phi_{0}(\boldsymbol{r}) e^{i \boldsymbol{Q} \cdot \boldsymbol{r}} d \boldsymbol{r}\right|^{2} .
$$

Besides the wave function of the initial bound state of the projectile $\phi_{0}$, it includes $\phi_{l j m}(E)$, the wave functions describing the $c-\mathrm{n}$ relative motion at energy $E$ in the continuum within partial wave $l j m$.

The expressions (4) and (6) explain qualitatively why similar cross sections are observed for breakup and elastic scattering [8]. Indeed both factorizations include the same point-like cross section, which describes most of the angular dependence of both cross sections. In addition, this analysis lead to the idea of the ratio method. Within the REB the ratio of Eqs. (4) and (6) is a mere ratio of two form factors and should be completely independent of the reaction mechanism. It should specially not depend on the $V_{c T}$ interaction that is the most uncertain optical potential in the reaction model.

\section{$4{ }^{11} \mathrm{Be}$ as a test case}

In order to test the ratio method, we confront the qualitative REB predictions with calculations of a fully dynamical reaction model, the DEA $[9,10]$, which does not rely on the adiabatic approximation, and which includes the interaction between the halo neutron and the target $V_{\mathrm{n} T}$. As a test case, we consider the collision of ${ }^{11} \mathrm{Be}$ on $\mathrm{Pb}$ at $69 \mathrm{AMeV}$, which correspond to the RIKEN experiment [2], with which the DEA is in excellent agreement [10]. Various tests have led us to choose instead of the elastic-scattering cross section (4), the summed cross section $d \sigma_{\text {sum }} / d \Omega$ to evaluate the ratio [6]. This cross section corresponds to all quasi-elastic processes: elastic and inelastic scattering as well as breakup to all energies $E$ in the $c$-n continuum. Using this cross section the REB prediction for the ratio becomes simply the form factor $\left|F_{E, 0}\right|^{2}(5)[5,6]$

$$
\frac{d \sigma_{\mathrm{bu}} / d E d \Omega}{d \sigma_{\text {sum }} / d \Omega} \stackrel{\text { REB })}{=}\left|F_{E, 0}\right|^{2} .
$$

The corresponding results are displayed in Fig. 1(left), where the breakup cross section (green dashed lines) and the summed cross section (ratio to Rutherford in blue dotted line) can be compared to each other. In agreement with the results of Ref. [8], they exhibit very similar patterns: Coulomb rainbow, oscillations at large angles... Their ratio [(8), red solid line] is hence very smooth, indicating that most of the sensitivity to the reaction mechanism is removed within this observable. This is further confirmed by the fact that the dynamical ratio is nearly superimposed onto its REB prediction (7) (thick grey line). Further tests have confirmed its independence to the reaction mechanism: the ratio computed for a carbon target is nearly identical to the one obtained on lead (see Fig. 1(right)) [5, 6]. Moreover, we have observed that the ratio can give access simultaneously to both the binding energy of the system and the orbital angular momentum of the halo neutron $[5,6]$. It is also sensitive to the radial wave function of the halo $[5,6]$. Valuable structure information can thus be extracted from this new observable. 

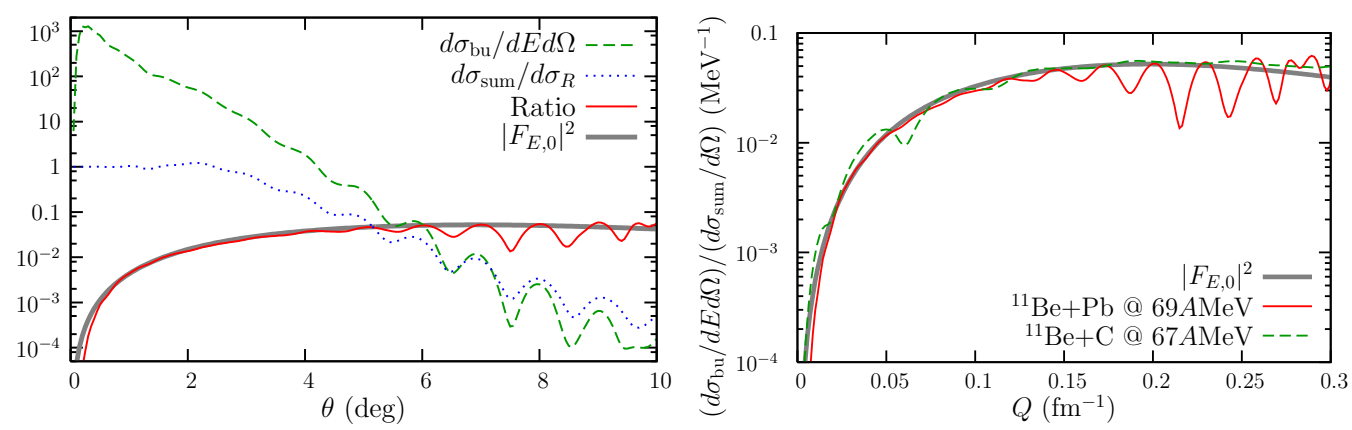

Figure 1. Left: ratio method illustrated on ${ }^{11} \mathrm{Be}$ impinging on $\mathrm{Pb}$ at $69 \mathrm{AMeV}$. The breakup angular distribution and the summed cross section are compared to each other. They exhibit very similar features, which vanish when taking their ratio. This ratio is in excellent agreement with the form factor predicted by the REB model. Right: comparison of the ratio obtained on $\mathrm{Pb}$ and $\mathrm{C}$ showing its independence on the reaction process $[5,6]$.

\section{Conclusion}

Halo nuclei have been discovered thanks to the development of RIBs [1]. Their very exotic structure has been the subject of many experimental and theoretical studies. It is studied mostly through reactions: elastic scattering, breakup ... Unfortunately the analysis of these reactions is hindered by dynamical effects and uncertainties in the reaction-model inputs. The ratio method suggests a new observable that is nearly independent of reaction processes and hence is less prone to model uncertainties. It consists of the ratio of angular distributions that is predicted by the REB to depend only on halo form factors [5, 6]. This prediction is analyzed within the state-of-the-art reaction model DEA for ${ }^{11} \mathrm{Be}$ impinging on $\mathrm{Pb}$ at $69 \mathrm{AMeV}$ and on $\mathrm{C}$ at $67 \mathrm{AMeV}$. It is shown to be nearly independent of the reaction mechanism and to be greatly sensitive to the projectile structure. In particular, both the halo binding to the core and its orbital can be precisely determined using the ratio [6]. Further tests should tell us whether it can be extended to other loosely-bound projectiles such as proton halos or two-neutron halo nuclei.

\section{References}

[1] I. Tanihata et al. Phys. Rev. Lett. 55, 2676 (1985).

[2] N. Fukuda et al. Phys. Rev. C 70, 054606 (2004).

[3] D. Baye and P. Capel, Breakup Reaction Models for Two- and Three-Cluster Projectiles in Cluster in Nuclei, Vol. 2, Lecture Notes in Physics 848, ed. C. Beck (Springer, Berlin, 2012), p. 121.

[4] P. Capel, H. Esbensen, and F. M. Nunes, Phys. Rev. C 85, 044604 (2012).

[5] P. Capel, R. C. Johnson, and F. M. Nunes, Phys. Lett. B705, 112 (2011).

[6] P. Capel, R. C. Johnson, and F. M. Nunes, Phys.Rev. C 88, 044602 (2013).

[7] R. C. Johnson, J. S. Al-Khalili, and J. A. Tostevin, Phys. Rev. Lett. 79, 2771 (1997).

[8] P. Capel, M. Hussein, and D. Baye, Phys. Lett. B693, 448 (2010).

[9] D. Baye, P. Capel, and G. Goldstein, Phys. Rev. Lett. 95, 082502 (2005).

[10] G. Goldstein, D. Baye, and P. Capel, Phys. Rev. C 73, 024602 (2006). 